\title{
Trasplante renal en paciente con pared abdominal compleja: síndrome de abdomen en ciruela pasa
}

\author{
Kidney transplant in a patient with a complex abdominal wall: \\ Prune belly syndrome
}

Javier Mozo ${ }^{1} \mathbb{D}$, Isabel Cristina Ángel ${ }^{2} \mathbb{D}$ MD, especialista en Cirugía general; cirujano de Trasplante renal, Hospital Universitario San Ignacio de Loyola, Bogotá, D.C.,
Colombia.
MD, residente de Cirugía general, Pontificia Universidad Javeriana, Hospital Universitario San Ignacio de Loyola, Bogotá, D.C.,
Colombia.

\section{Resumen}

Introducción. Se presenta un paciente con falla renal crónica secundaria a hidroureteronefrosis bilateral, con antecedente de síndrome de abdomen en ciruela pasa (prune belly), en quien se realiza trasplante renal.

El objetivo de este reporte de caso es exponer una anomalía congénita, con baja incidencia mundial y en menor proporción asociado a trasplante renal, con énfasis en la dificultad para la técnica quirúrgica dada por la hipoplasia de los músculos de la pared abdominal.

Métodos. Revisión de Historia clínica, Consentimiento Informado. Búsqueda de la literatura.

Caso clínico. En cirugía se identifica ausencia de músculos de la pared abdominal y fibrosis de las venas Iliaca externa y común derecha. Se realiza abordaje bajo técnica de Gibson contralateral, con trasplante renal sin complicaciones. Presentó adecuada evolución en seguimiento ambulatorio.

Discusión. El síndrome de abdomen en ciruela pasa (prune belly) es una anomalía congénita de baja incidencia, con pocos reportes asociados a trasplante renal. El reto quirúrgico está dado por la ausencia de músculos de la pared abdominal, que aumentan los riesgos por posibles complicaciones, como la lesión de los vasos epigástricos inferiores. Para el cierre de pared abdominal en trasplante renal, se describe en dos planos, que en este caso se realiza sobre fascia muscular y piel. Aun siendo un reto, el paciente presenta adecuada evolución postoperatoria.

Palabras clave: trasplante; trasplante de riñón; insuficiencia renal crónica; síndrome del abdomen en ciruela pasa; pared abdominal.

Fecha de recibido: 11/06/2019 - Fecha de aceptación: 25/10/2019

Correspondencia: Isabel Cristina Ángel, Carrera 7 \# 32-48, Bogotá, D.C., Colombia. Teléfono: 3113286437

Correo electrónico: isc.angel02@gmail.com

Citar como: Mozo J, Ángel IC. Trasplante renal en paciente con pared abdominal compleja: Síndrome de abdomen en ciruela pasa. Rev Colomb Cir. 2020;35:520-5. https://doi.org/10.30944/20117582.788

Este es un artículo de acceso abierto bajo una Licencia Creative Commons - BY-NC-ND https://creativecommons.org/licenses/by-ncnd/4.0/deed.es 


\begin{abstract}
Introduction. We present a patient with chronic renal failure secondary to bilateral hydroureteronephrosis, with a history of prune belly syndrome, who underwent a kidney transplant. The objective of this case report is to expose a congenital anomaly, with a low worldwide incidence and to a lesser extent associated with kidney transplantation, with emphasis on the difficulty for the surgical technique due to the hypoplasia of the abdominal wall muscles.
\end{abstract}

Methods. Review of clinical history, Informed Consent. Literature search.

Clinical case. Absence of abdominal wall muscles and fibrosis of the external iliac veins and right common veins were identified intraoperatively. An approach was performed under the contralateral Gibson technique, with uncomplicated kidney transplantation. He presented adequate evolution in outpatient follow-up.

Discussion. Prune belly syndrome is a low-incidence congenital anomaly, with few reports associated with kidney transplantation. The surgical challenge is given by the absence of abdominal wall muscles, which increases the risks due to possible complications, such as injury to the inferior epigastric vessels. For the closure of the abdominal wall in kidney transplantation, it is described in two planes, which in this case is performed on muscle fascia and skin. Despite being a challenge, the patient has adequate postoperative evolution.

Key words: transplantation; kidney transplantation; renal insufficiency, chronic; prune belly syndrome; abdominal wall.

\section{Introducción}

El síndrome de abdomen en ciruela pasa (prune belly) es una enfermedad congénita, predominante en pacientes masculinos, donde ocurre displasia renal, asociada a dilatación tubular y diferenciación incompleta de nefronas, glomeruloesclerosis y fibrosis intersticial, ligado a atrofia tubular. Clínicamente se identifica por la triada de hipoplasia de musculatura abdominal, anomalías del tracto urinario y criptorquidia bilateral. Tiene una incidencia de hasta 3,8 casos por cada I0o.0oo nacidos vivos '; en Colombia, se han reportado por medio del Estudio Colaborativo Latino Americano de Malformaciones Congénitas (ECLAMC) ${ }^{2}, 2$ casos en Cali y 6 en Bogotá del 2008 al 2017.

El trasplante renal en pacientes con anomalías congénitas del tracto urinario conlleva un reto quirúrgico, que representa desafíos complejos en el manejo de la pared abdominal y del sistema de excreción urinario ${ }^{3,4}$. El $30 \%$ de los pacientes con síndrome de abdomen en ciruela pasa (prune belly) en la adolescencia presentan falla renal asociada a displasia, pielonefritis recurrente o nefropatía obstructiva ${ }^{5-7}$. El primer caso de trasplante renal en un paciente con síndrome de abdomen en ciruela pasa (prune belly) se reportó en I976, demostrando la necesidad de preservar la función vesical y la posibilidad de realizar este procedimiento con buena función del injerto, con adecuados desenlaces clínicos a corto, mediano y largo plazo $^{8-10}$.

El objetivo de este reporte de caso es exponer una anomalía congénita con pocos reportes en Colombia, y en menor proporción asociada a trasplante renal ${ }^{2, \mathrm{II}}$, por lo que se describe el caso de forma integral, con énfasis en las alteraciones de la pared abdominal.

\section{Caso clínico}

Paciente masculino de 29 años, con antecedente de compromiso renal desde los 3 años de edad, con deterioro progresivo hasta enfermedad renal crónica (ERC) estadio 5, en hemodiálisis interdiaria, con necesidad de cateterismo vesical intermitente, asociado a síndrome de abdomen en ciruela pasa (hidroureteronefrosis bilateral), sin procesos infecciosos recientes ni complicaciones secundarias a la diálisis. Adi- 
cionalmente, con antecedentes relevantes de epilepsia refractaria, con lobectomía del lóbulo temporal derecho hace 4 años, hiperparatiroidismo secundario, orquidopexia bilateral, colecistectomía y fístula arterio-venosa en el brazo derecho. Entre los últimos estudios diagnósticos se destaca una ecografía Doppler de vasos abdominopélvicos (2016) sin evidencia de trombos o placas ateromatosas en arterias o venas y un ecocardiograma (marzo de 20I8) con leve hipertrofia concéntrica del ventrículo izquierdo y fracción de eyección de $68 \%$.

En noviembre de 2018 se realiza trasplante renal con donante cadavérico, considerado de bajo riesgo inmunológico, por panel reactivo de anticuerpos de o \%, con compatibilidades aceptables para moléculas de Clase I y Clase II, y mismatch de 4/I (tabla I), y de riesgo intermedio para infección por citomegalovirus. Se registró isquemia fría de II:Io minutos e isquemia caliente de 40 minutos.

Se realiza previamente paso de catéter venoso central, terapia de inducción con Basiliximab y antibiótico profiláctico (Cefazolina 2 gramos). Riñón izquierdo de donante con dos arterias renales en parche único, vena renal única, y uréter único. En el receptor, hallazgos de pared abdominal con aponeurosis, pero sin músculos rectos y oblicuos de lado derecho, venas iliaca externa y común derecha fibrosadas, por lo que se decide realizar abordaje de Gibson izquierdo, observando hipoplasia de músculos rectos y oblicuos, vena iliaca externa izquierda permeable, arteria iliaca de buena calidad y vejiga normal. Tiempo quirúrgico de 4 horas, presión arterial media (PAM) en reperfusión de $90 \mathrm{mmHg}$, líquidos administrados de 2500 cc de cristaloides, uso de Manitol con diuresis inmediata, sangrado

Tabla 1. Antígenos leucocitarios en humanos - HLA (Human leukocyte antigen).

\begin{tabular}{cccc}
\hline HLA & \multicolumn{2}{c}{ Clase I } & Clase II \\
\hline Donante & A02/A02 & B18/B51 & DR4/DR4 \\
\hline Receptor & A03/A03 & B18/B57 & DR04/DR11 \\
\hline
\end{tabular}

intraoperatorio de $200 \mathrm{cc}$, cierre de pared abdominal en un solo plano y cierre de piel.

En el postoperatorio presentó función retardada del injerto y un pequeño hematoma del polo renal superior, que no requirió manejo adicional. Cursó con sepsis de origen urinario por pielonefritis del injerto (por E. coli BLEE), con falla ventilatoria hipoxémica, falla cardiaca y estatus convulsivo, resueltos.

Con adecuada evolución al salir de la Unidad de Cuidados Intensivos, dándosele de alta después de 36 días de hospitalización, sin signos de respuesta inflamatoria sistémica, y heridas con adecuada cicatrización (figura I). Función adecuada y estable del injerto renal, paraclínicos de seguimiento con ascenso de la hemoglobina, sin leucocitosis, azoados y electrolitos estables. En seguimiento por consulta externa, con adecuada evolución clínica, continúa realizándose cateterismos vesicales 4 veces al día, sin complicaciones.
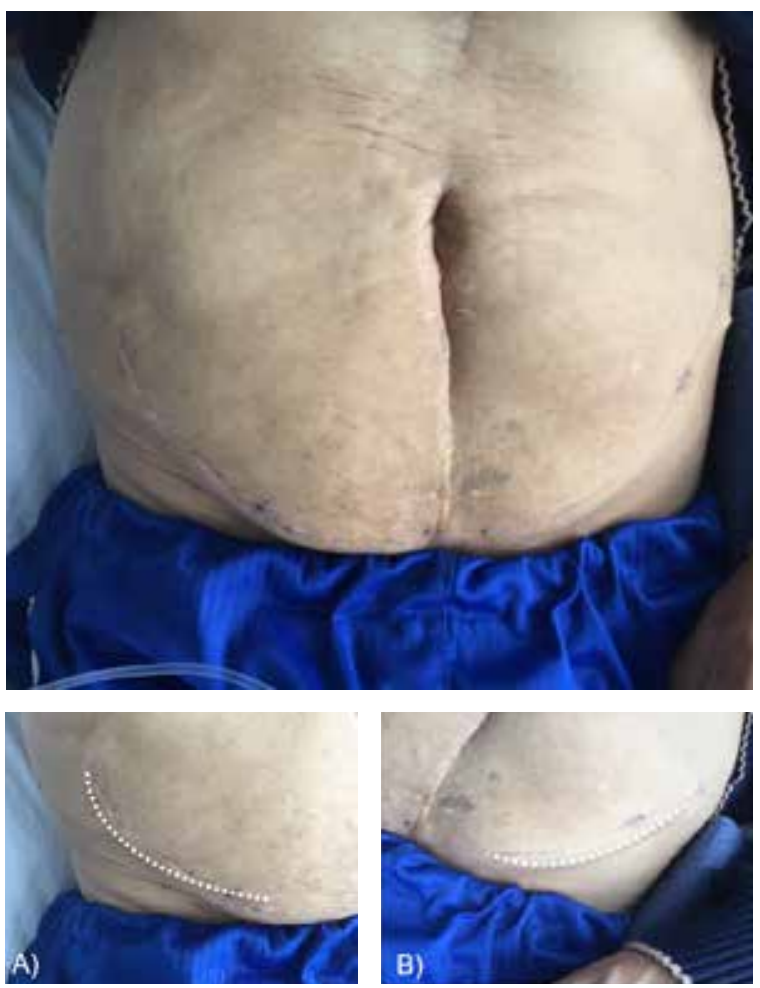

Figura 1. Apariencia de pared abdominal en ciruela pasa por hipoplasia de los músculos de pared abdominal. A) Incisión de Gibson derecha. B) Incisión de Gibson izquierda. 


\section{Discusión}

El pronóstico a largo plazo de los pacientes con síndrome de abdomen en ciruela pasa (prune belly) se ve afectado por los problemas asociado a la enfermedad renal, y posteriormente, a la necesidad de trasplante renal. Yalcincaya y colaboradores ${ }^{9}$ reportaron que más del $40 \%$ de los pacientes con síndrome de abdomen en ciruela pasa, requieren diálisis antes de los 5 años de edad, y casi el $20 \%$ inician a los 6 meses de vida, determinando la obstrucción del tracto urinario como la causa predominante de la falla renal.

En la literatura se encuentran muy pocos casos de trasplante cadavérico en pacientes con enfermedad congénita del tracto urinario $(5 \mathrm{a}$ 9 pacientes), específicamente en pacientes con síndrome de abdomen en ciruela pasa ${ }^{9}$, a pesar de que es una opción, con resultados similares respecto a otras anomalías congénitas del riñón y del tracto urinario; sin afección negativa en los resultados postrasplante y sin evidencia de falla del injerto en Io años, según lo reportado en el estudio de Fontaine ${ }^{8}$.

El síndrome de abdomen en ciruela pasa (prune belly) conlleva severas comorbilidades que afectan la supervivencia del injerto, de las cuales hay limitada información registrada. Las causas de muerte asociadas, en orden de frecuencia, son las infecciones y las enfermedades cardiovasculares; evento que presenta el paciente y resuelve durante la hospitalización, con buena evolución.

Es importante aclarar dentro de la técnica quirúrgica de trasplante renal la localización habitual del trasplante, el cual es heterotópico, y se realiza inicialmente en la fosa iliaca derecha, debido a que los vasos iliacos en esa localización son más superficiales, lo que facilita la reconstrucción vascular. Igualmente se recomienda la ubicación del riñón (derecho o izquierdo), en la fosa iliaca contralateral, para tener una exposición anterior de la pelvis renal y el uréter, facilitando la reconstrucción del tracto urológico ${ }^{12}$ (Figura 2).

Se describe la incisión oblicua de RutherfordMorris, que es una incisión curvilínea paralela, $2 \mathrm{~cm}$ sobre el ligamento inguinal, finalizando en

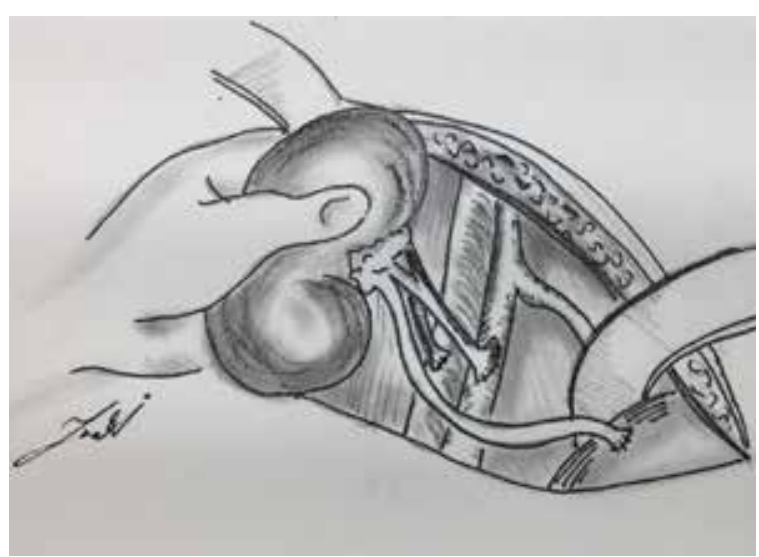

Figura 2. Representación esquemática de la localización del trasplante renal, permitiendo identificar la anastomosis de la vena, la arteria y el uréter del injerto, a la vena iliaca, la arteria lliaca y la vejiga del paciente.

la espina iliaca anterosuperior de la cresta iliaca ${ }^{\text {I2}}$; la incisión se extiende medial hasta la hoja del recto abdominal, para permitir la retracción o división del resto de músculos de la pared abdominal, y posteriormente la exposición de la vejiga. Para la exposición del peritoneo, el músculo oblicuo interno y el trasverso se dividen con electrocauterio, o la confluencia entre los oblicuos y la hoja del recto es dividida medialmente, para después ligar y dividir, de ser necesario, los vasos epigástricos inferiores.

Otra opción es la incisión de Gibson, descrita como incisión en "palo de hockey", que se extiende desde los $2-3 \mathrm{~cm}$ de la espina iliaca anterosuperior y el ligamento inguinal, hasta el borde extremo del musculo recto homolateral, incidiendo el tejido celular subcutáneo y el oblicuo mayor, con posterior sección del oblicuo menor y el transverso, en dirección de sus fibras respectivamente. Luego se realiza disección digital de la fascia transversalis y del peritoneo, hasta la línea media ${ }^{13}$.

Todo esto con el fin de preparar el plano quirúrgico, que permite exponer la fascia transversalis y peritoneo, el cual se rechaza hacia medial y superior, para exponer los vasos iliacos y el músculo psoas, en un abordaje extra y retroperitoneal ${ }^{12}$. 
El reto quirúrgico en este reporte de caso está dado por la ausencia de músculos de la pared abdominal, que dificultaban la adecuada referenciación anatómica en el abordaje, dejando expuesto a los riesgos y las complicaciones descritas, como la lesión de los vasos epigástricos inferiores, así como la necesidad disección cuidadosa, con el fin de evitar daño a estructuras vasculares o intestinales que, por la agenesia de la pared, se encuentran más superficiales.

El cierre de la pared abdominal en trasplante renal se describe en dos planos, inicialmente los músculos transverso y oblicuo interno, seguido del músculo oblicuo externo y terminando con sutura intradérmica de la piel. Es importantedestacar los riesgos del cierre de la pared abdominal ${ }^{14}$, entre ellos, el síndrome de compartimiento abdominal o la compresión anteroposterior, que puede comprometer el lecho vascular, llevando a estasis y trombosis del injerto.

De igual forma, se describe el reposicionamiento del injerto, por cambio en su eje, permitiendo la rotación del mismo ${ }^{15}$. Para este último caso, en la literatura se discute también la abdominoplastia, con el fin de proteger de la posibilidad de torsión del injerto ${ }^{4}$, con excelentes resultados cosméticos, e incluso, mejoría en la función vesical e intestinal ${ }^{5}$. Sin embargo, en el artículo de Kamel y colaboradores ${ }^{3}$, con seguimiento a 9 años, no encontraron diferencias estadísticamente significativas de torsión del injerto, aún en técnicas como la nefropexia en los pacientes trasplantados.

Por último, los pacientes con síndrome de abdomen en ciruela pasa (prune belly) y trasplante renal, son más susceptibles a presentar complicaciones asociadas a sus patologías de base, como falla cardiaca, estatus convulsivo, infección y hematoma alrededor del injerto, que resuelven con manejo médico intrahospitalario ${ }^{3}$. De igual forma, la presencia de estrechez uretral con necesidad de cateterismos intermitentes se asocia a una vejiga disfuncional en estos pacientes, como nuestro paciente, quien requiere cateterismos vesicales intermitentes ${ }^{16}$.
El caso que exponemos presenta un postoperatorio con complicaciones contempladas en la literatura ${ }^{17,18}$, que se resuelven durante la estancia hospitalaria, con adecuada evolución clínica ambulatoria, con una tasa de filtración glomerular actual de 71,03 (Cockcroft) y realizando cateterismos intermitentes. Los distintos abordajes quirúrgicos de la incisión inicial, así como el cierre de pared abdominal, se convirtieron en un reto, que se logró resolver con resultados clínicos satisfactorios, teniendo como resultado final que el síndrome de abdomen en ciruela pasa (prune belly) no afectó el trasplante renal ${ }^{19}$.

\section{Conclusión}

El Síndrome de abdomen en ciruela pasa (prune belly) es una anomalía congénita de baja incidencia, con muy pocos casos reportados en Colombia. También, mundialmente son pocos los reportes de esta patología asociada a trasplante renal. Se expone el caso de un paciente sometido a trasplante renal, en quien se resalta la agenesia de la pared abdominal como reto quirúrgico, al exponer a riesgo de lesión las estructuras anormalmente más superficiales, por no contar con la disposición normal de los músculos de la pared abdominal. Esto, asociado al evento de fibrosis de la vena iliaca derecha, que obliga a un abordaje contralateral en el mismo paciente, lo que llevó a un mayor tiempo quirúrgico, doble incisión, y cierre de fascia y piel de manera no convencional.

\section{Cumplimiento de normas éticas}

Consentimiento informado: Este estudio es una revisión de historia clínica retrospectiva, y por lo tanto se considera sin riesgo para el paciente. Se obtuvo consentimiento informado por parte del paciente.

Conflicto de intereses: Ninguno reportado por los autores.

Financiación: este trabajo fue autofinanciado. 


\section{Referencias}

I. Routh JC, Huang L, Retik AB, Nelson CP. Contemporary epidemiology and characterization of newborn males with prune belly syndrome. Urology. 20I0;76:44-8. https://doi.org/IO.IOI6/j.urology.2009.12.072

2. ECLAMC - Estudio colaborativo latinoamericano de malformaciones congénitas - programa de vigilancia y seguimiento de defectos congénitos Bogotá-Cali. Pontificia Universidad Javeriana. Prune Belly (2008-2017). Fecha de consulta: 2I de enero de 20I9. Disponible en: http://www.anomaliascongenitas.org

3. Kamel MH, Thomas AA, Al-Mufarrej FM, O'Kelly P, Hickey DP. Deceased-donor kidney transplantation in prune belly syndrome. Urology. 2007;69:666-9. https://doi.org/IO.IoI6/j.urology.2007.0I.0I5

4. Bagga HS, Lin S, Williams A, Schold J, Chertack N, Goldfarb D, et al. Trends in renal transplantation rates in patients with congenital urinary tract disorders. J Urol. 2016;195:1257-62. https://doi.org/Io.IoI6/j.juro.2015.IO.004

5. Fusaro F, Zanon GF, Ferreli AM, Giuliani S, Zacchello G, Passerini-Glazel G, et al. Renal transplantation in prune belly syndrome. Transpl Int. 2004;17:549-52. https://doi.org/I0.IO07/sool47-004-0759-7

6. Smith CA, Smith EA, Parrott TS, Broecker BH, Woodard JR. Voiding function in patients with the prune belly syndrome after Monfort abdominoplasty. J Urol. I998;159:I675-9 https://doi.org/IO.I097/00005392-I99805000-00089

7. Seidel NE, Arlen AM, Smith EA, Kirsch AJ. Clinical manifestations and management of prune-belly syndrome in a large contemporary pediatric population. Urology. 20I5;85:2II-5.

https://doi.org/IO.IOI6/j.urology.20I4.09.029

8. Fontaine E, Salomon L, Gagnadoux M, Nuudet P, Broyer $M$, Beurton D. Long-term results of renal transplantation in children with prune-belly syndrome. J Urology. 1997;I58(3 Pt I):892-4.

9. Yalcinkaya F, Bonthuis M, Erdogan BD, van Stralen KJ, Baiko S, Chehade H, et al. Outcomes of renal replacement therapy in boys with prune belly syndrome: findings from the ESPN/ERA-EDTA registry. Pediatr Nephrol. 2018;33:117-24. https://doi.org/IO.IOO7/soo467-0I7-3770-9
Io. ShenaskyII J, Whelchel J. Renal transplantation in prune belly syndrome. J Urol. 1976;II5:II2-3. https://doi.org/IO.IOI6/Soo22-5347(I7)59088-6

II. Guerrero AF, Cuadros CA, Archila DC, Beltrán SM, Cuadros GA. Síndrome de prune belly: Presentación de un caso y revisión de la literatura. Revista Salud UIS. 20I0:42:78-85.

I2. Barry JM, Morris PJ. Surgical techniques of renal transplantation. 6th ed. In: Knechtle S, Morris PJ, editors. Principles and practice: Kidney transplantation. London, UK: Saunders; 2008. p. 158-I65.

13. Fayad E, López M. Anatomía quirúrgica y vías de acceso en urología. Módulo I - Fascículo 2. En: Programa de actualización continua y a distancia en urología. Buenos Aires: Sociedad Argentina de Urología; 200I. p. I-9. Fecha de consulta: I7 de marzo de 20I9. Disponible en: https://www.sau-net.org/comites/educacion/fasciculos/ anatomia2.pdf

I4. León- Hernández MA, Acosta-León J, Cárdenas-Camarena L. Síndrome de prune belly: Cirugía para mejoría estética y reconstrucción abdominal. Cir. plást. iberolatinoam. 20I5;41:I75-8. https://doi.org/IO.432I/So376-78922015000200008

15. Halawa A. Abdominal wall closure of renal transplant recipients: An undermined challenge. Int J Organ Transplant Med. 20I0;1:73-6.

I6. Banno T, Kakuta Y, Unagami K, Sakoda A, Okumi M, Ishida $\mathrm{H}$, et al. Living-related kidney transplantation with catheterizable urinary conduit in prune belly syndrome: A case report. Inter J Surg Case Rep. 2018;5I:150-3. https://doi.org/IO.IOI6/j.ijscr.20I8.08.022

17. Stenzl A, Fuchs GJ. Case history of a prune-belly syndrome with extracorporeal shock wave lithotripsy treatment of allograft nephrolithiasis. Urol Int. 1989;44:I06-9. https://doi.org/I0.II59/00028I48I

I8. Reinberg Y, Manivel JC, Fryd D, Najarian JS, Gonzalez R. The outcome of renal transplantation in children with the prune belly syndrome. J Urol. I989;I42:I54I-2. https://doi.org/IO.IOI6/s0022-5347(I7)39I56-5

19. Marchal S, Kalfa N, Iborra F, Badet L, Karam G, Broudeur L, et al. Long-term outcome of renal transplantation in patients with congenital lower urinary tract malformations: A multicenter study. Transplantation. 2020;104:I65-7I.

https://doi.org/I0.I097/TP.oooooooooooo2746 